\title{
NeW Insights FROM Old DATA: MUlTimodal ClassificAtion of SCHIZOPHRENIA USING AUtOMATED DeEP LEARNING CONFIGURATIONS
}

\author{
A PREPRINT \\ Gagana B \\ AI Without Borders \\ gaga@wob.ai
}

January 5, 2021

\begin{abstract}
Schizophrenia is a heterogeneous cognitive disorder where clinical classification is challenging because of the lack of well-established, non-invasive diagnoses biomarkers. There is, hence, a need for objective systems that can classify Schizophrenia despite challenges such as overlapping symptomatic factors, diverse internal clinical manifestations, and complex diagnostic process leading to delayed treatment. Thus, experimentation with automated machine learning architectural frameworks $(\mathrm{Au}-$ toML) is presented in order to handle multimodal Functional Network Connectivity(FNC) and Source Based Morphometry(SBM) features based on functional magnetic resonance imaging(fMRI) and structural magnetic resonance imaging(sMRI) components respectively. On evaluating the resultant AutoML models with respect to approximately 280 machine learning architectures on the Overall AUC metric, the former outperforms the latter despite remarkable limitations including complex high dimensional feature space with very little data.
\end{abstract}

Keywords Schizophrenia $\cdot$ AutoML $\cdot$ fMRI $\cdot$ sMRI $\cdot$ Functional Network Connectivity(FNC) - Source Based Morphometry $(\mathrm{SBM}) \cdot$ Neural Architecture Search

\section{Introduction}

\subsection{Schizophrenia}

Schizophrenia is a mental fragmentation disorder[55] that is predominantly characterized by distorted perception of reality, hallucinations or delusions, and thought disorganization[16]. Diagnosis relies on the use of subjective processes of elimination due to the lack of well-established, non-invasive diagnoses clinical biomarkers[46], although there has been extensive interest in the medical community for its' development[29][30] aimed at aiding diagnostic, prognostic and theranostic research[5].

The condition, however, with its elusive pathophysiology[39] is known to be surprisingly common ${ }^{1}$. The differential diagnosis of schizophrenia is however challenging specially while discriminating schizoaffective disorder from other closely related mental illnesses including clinical depression[46](specially in the premorbid and prodromal phases of preschizophrenia), bipolar I disorder[46][47] and attention-deficit/hyperactivity disorder(ADHD)[48]. Schizophrenics may thus receive multiple diagnosis based on appearance of symptoms which greatly influence treatment practices[31].

Schizophrenia is also associated with diverse internal clinical manifestations including abnormalities in anatomy of limbic-cortical systems[3], dysfunction in medial temporal (particularly hippocampal region) and lateral temporal

\footnotetext{
${ }^{0}$ Accepted as workshop contribution at Women in Machine Learning (WiML) co-located with ICML 2020. Related code can be found at: https://github.com/GaganaB/AutoML

https://www.health.harvard.edu/a_to_z/schizophrenia-a-to-z
} 
lobe structures[40] and synaptic pruning[56]. However, at a neuronal level: the superior temporal gyrus appears to be less volumetric which negatively correlates with severity of corresponding abnormalities - hallucinations and thought disorders[2]. Schizophrenia is hence classified across a spectrum that ranges from attenuated schizoid and mild schizotypal personality to the most severe and debilitating forms[32].

Factors that potentially affect clinical assessments are:

(a) Prolonged use of potent stimulants like Methamphetamines or hallucinogenic drugs like Lysergic acid diethylamide(LSD) that can impart schizophrenia-like symptoms[8].

(b) Schizophrenia is known is to run in families[36] but there looms uncertainty concerning the degree of genetic contribution to the phenotypic variance of the disease[57]. These observations have led to scientific speculations that interactions between internal genetic components and external environments could trigger the disease[10].

(c) Complications during pregnancy, childhood trauma, fetal growth retardation[58], inflammation of the brain, head injury, emotional consequences of imprisonment, and hypoxia[49] are also identified as activation points.

(d) Research also further examines the role of external factors like employability and financial status of the patient as well as age and workload of the treating physician in the patient-physician relationship through the continuity of care (COC) metric[7].

To prevent any interference with diagnosis and subsequent treatment, diagnostic procedures rely on metrics including DUP (Duration of untreated psychosis) and DPIT (Delay in intensive treatment) which, in case of Schizophrenia could fail to account for the neurodevelopmental disturbances resulting in cognitive deficits before the onset of psychotic symptoms. The metrics measure a median of around twenty four weeks[35] which further highlight the need for early clinical or technological interventions[34].

\subsection{Automated Machine Learning}

With tremendous growth in the amount of data and complexity, designing machine learning systems is expertise intensive and tuning machine learning systems is time consuming. Automated machine learning (AutoML) attempts to automate these pipelines eliminating the confusion of whether certain models really perform better or were simply better tuned.

The generic workflow of a typical AutoML framework is as follows: The training data is fed into a system that automatically generates model architecture which has associated configurations that are tunable and hyperparameters which can be shipped for further predictions to be made on new data.

But the comparison of various AutoML frameworks and configurations is often tricky because[6]:

(a)Blackbox systems in the background differ in their optimization methodologies, pipeline generation techniques, internal architectures and processing procedures while operating on similar data[13]. The results may also vary on other parameters including training time, memory and computational resources.

(b) Reruns of the same configurations on data often yield different results. This tends to affect result reproducibility and complicate evaluation process.

(c) Some frameworks automate the entire pipeline seamlessly while others tend to rely on external engineering techniques. In the latter case, performance evaluation is not a wholesome indicator of the framework's capabilities. Across the machine learning pipeline, most of the AutoML tools have fairly similar processes with respect to data cleaning, data coding, metric selections but differ in their algorithm selection, parameter optimisation and post processing abilities.

Multiple surveys of automated machine learning frameworks focus on evaluating tools on various data[6][13][14][15]. But investigations are limited on the performance of various configurations of these tools on similar data. Although current surveys do help perform guided research to some extent, the need for trial and error evaluation of tools is not completely eliminated. There are no universal standardised configuration benchmarks and that necessitates trial and error evaluation in order to select the right tools and corresponding configurations on any given data. In this context, automated machine learning has not been explored extensively and prior research has shown that automated frameworks and tools do not necessarily perform better than machine learning algorithms[6]. This paper hence explores the use of automated machine learning (AutoML) on multimodal schizophrenia data to outperform classification algorithms.

This paper aims to study the architectures and corresponding performances of AutoML on multimodal data, with specific metrics particularly in healthcare. Also, as such, classification of individual subjects based on observed behavioral features[12] is more challenging than reporting group differences via voxel based approaches[1]. Some inconsistencies resulting from data representativeness heuristics[27] reflect the true reality of neurological research thus making this data a perfect challenge for AutoML. 
A PREPRINT - JANUARY 5, 2021

\section{Background and Related work}

This section is intended to reflect on past literature in both biomedical research in Schizophrenia classification as well as in automated methodologies applicable to healthcare.

\subsection{Current Clinical Detection Processes}

Most of the defining attributes of schizophrenia are based on inferences drawn from self reported subjective experiences (along with but not limited to psychiatric status observation of family and personal history - evaluation of demeanor, appearance, thought process about delusions, hallucinations, and potential for violence or suicide) owing to the complexity of the disorder and the ambiguity in its diagnostic concept itself. The detection process involves a comprehensive medical examination with treatment regimen to reduce appearance of symptoms[42].

\subsection{Exploration of AutoML for healthcare systems}

The evolution of machine learning has contributed significantly to the healthcare industry through image detection, classification, and segmentation tasks but with respect to interest in mental health, there has been massive transformation specially with non-invasive neuroimaging[18]. For schizophrenia, in particular, machine learning methods[43] have been explored for diagnostics and classification from structural MRI[44] and functional MRI features[45], EEG signals[11] and resting EEG streams[4].

However, with respect to AutoML, the current research portfolio is sparse. One of the open benchmarks involving AutoML tools[6] suggests that:

(i) AutoML does not perform better than untuned machine learning models(random forests).

(ii) There is no AutoML system which consistently outperforms all others.

For blood transfusion in particular, $\mathrm{H} 2 \mathrm{O}$ performs best as compared to other AutoML frameworks.

Another similar study[15] used AutoML to classify the following healthcare datasets:

(a) Breast cancer(OpenML dataset ID 15) where ATM[26] seemed to perform the best(Average accuracy: 0.98474).

(b) Diabetes(OpenML dataset ID 37) where hyperopt seemed to perform the best(Average accuracy: 0.7996).

(c) Gene splice data(OpenML dataset ID 46) where hyperopt seemed to perform the best(Average accuracy: 0.9654).

(d) Blood-transfusion data(OpenML dataset ID 1464) where RoBO seemed to perform the best(Average accuracy: 0.8076).

(e) EEG eye state data(OpenML dataset ID 1471) where SMAC seemed to perform the best(Average accuracy: 0.9741).

\section{Data Description}

Owing to the adversities articulated in Section 1, there is a need to design automated processes that can classify schizophrenia but the technical challenge is to optimally combine the resting-state functional MRI and the gray-matter concentration information of structural MRI to automatically predict diagnosis labels and optimize performance. Schizophrenia is believed to be better characterized by the effective relationship between functional and structural data[27] which further assists in the understanding of pathology of the brain in general and specifically, contributes to the hunt for biomarkers.

\subsection{Data Collection}

The training data has 378 functional network connectivity features and 32 source based morphometry features with information on 144 subjects(75 healthy controls and 69 schizophrenic patients) acquired using a 3T Siemens Trio MRI scanner with a 12-channel head coil[27].

\subsection{Data Preprocessing}

SPM5 standard preprocessing technique ${ }^{2}$ was employed to eliminate volumes leading to equilibration effects, image realignment, and correction of discontinuities in slice timings. Data was further spatially normalized, re-scaled to a mean of 100 and analyzed with spatio-temporal regression group independent component analysis (GICA)[27][60]. Independent component analysis further extracts artifacts embedded in the data from the preprocessed output using

\footnotetext{
$\sqrt[2]{\text { www.fil.ion.ucl.ac.uk/spm/software/spm5 }}$
} 
bioRxiv preprint doi: https://doi.org/10.1101/2020.11.02.364976; this version posted January 5, 2021. The copyright holder for this preprint (which was not certified by peer review) is the author/funder, who has granted bioRxiv a license to display the preprint in perpetuity. It is made available under aCC-BY-NC-ND 4.0 International license.

A PREPRINT - JANUARY 5, 2021

spatio-temporal regression[37]. The structural and functional data were then mapped into the space of a healthy control baseline dataset from which features were derived.

In the resultant data features, it was observed that there is little correlation between the structural and functional components but the groups amongst themselves are highly correlated.

\subsection{Data Features}

\subsubsection{Functional MRI : Functional Network Connectivity features}

These functional feature modality values describe the overall connection levels between pairs of independent brain maps over time. The connectivity pattern between brain networks (also called synchronicity between brain areas) is mapped using the functional network connectivity features where the time courses indicate the activity level of the corresponding brain map at that point using the Pearson correlation values and hence, allows exploration of the neural interaction properties within the brain. Disruptive patterns are known to be indicative of schizophrenia[59]. The FNC matrix is symmetric and only the unique values are retained as features in this classification context[20].

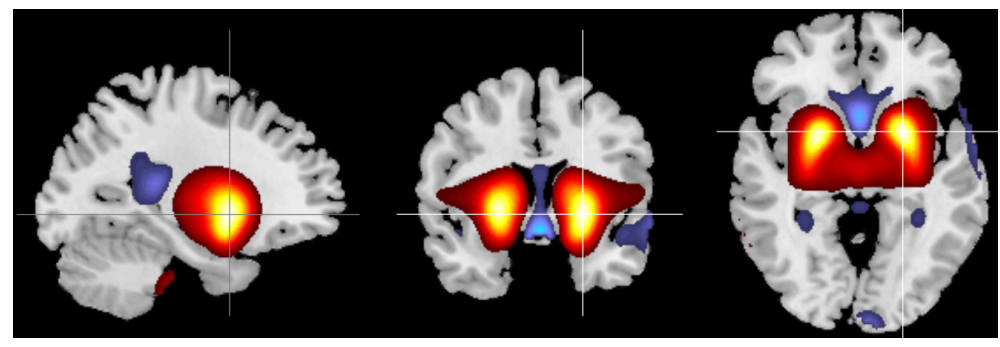

Figure 1: Visualization of FNC features

Observations: The data distribution of the FNC features is near-normal as shown in Figure 2.

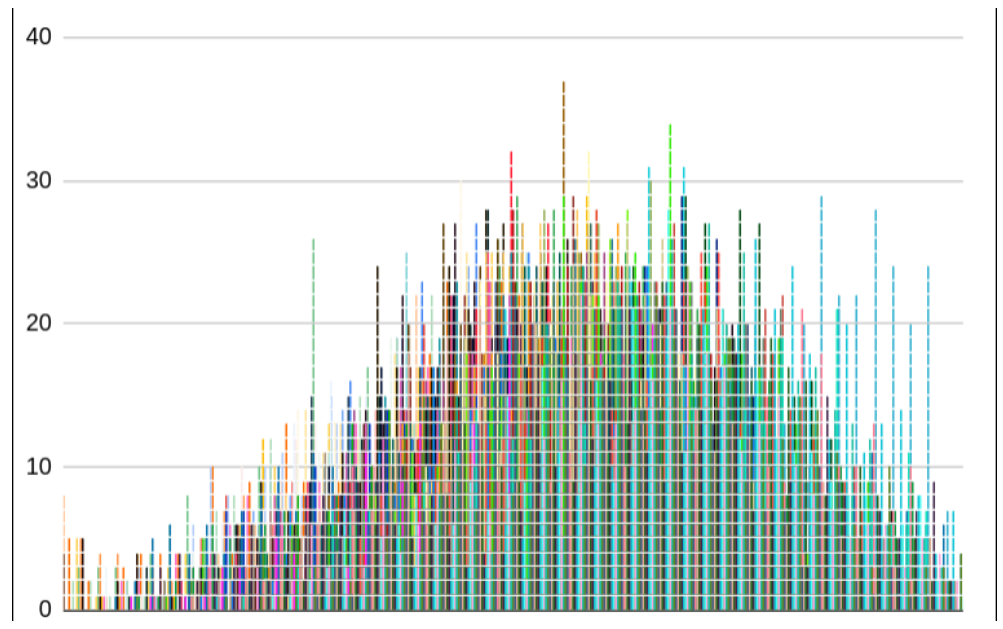

Figure 2: Visualisation of distribution of FNC features[20]

\subsubsection{Structural MRI: Source Based Morphometry features}

Source based morphometry loadings[21] correspond to the standardised weights of all subjects with respect to the level of independent component analysis(ICA) brain maps derived from grey-matter concentration. The activity of the signal processing is further indicative of the computational power available in that region of the brain that is monitored along with the tissue density of grey matter, white matter and cerebrospinal fluid in the region[38][50][51]. A near zero structural modality feature value for given ICA derived brain maps is indicative that the brain regions in that subject are lowly present and hence, suggestive of schizophrenia[52][53][54].

Observations: The data distribution of the SBM features appears normal as shown in figure 4. 


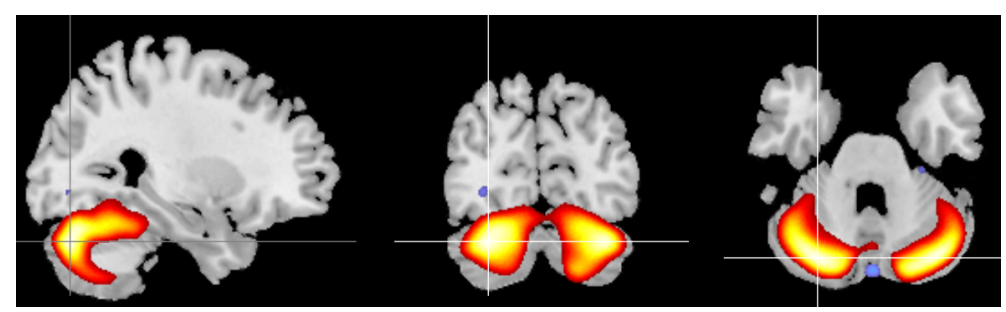

Figure 3: Visualization of SBM features[21]

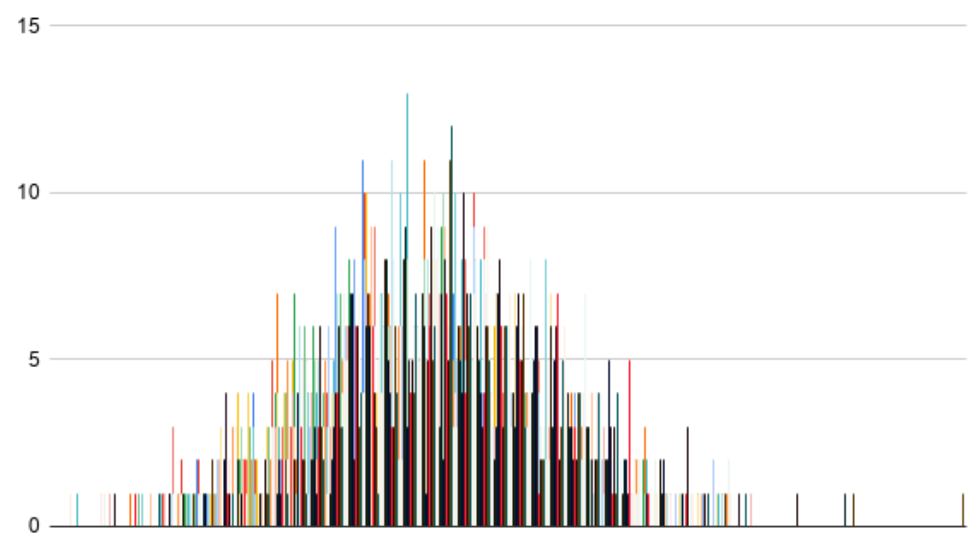

Figure 4: Visualisation of distribution of SBM features

\section{Methodology}

This section deals with two aspects: (a) Description of machine learning algorithms against which automated methods are compared and

(b) The following open-source automated machine learning frameworks: Ludwig[22], $\mathrm{H} 2 \mathrm{O}$ [23], AutoGluon-Tabular[24], MLBox[25], ATM[26] and AutoML-gs have been evaluated. In order to analyse the competency of the AutoML framework itself, pure automated approaches were used with no manual interventions in feature engineering, data cleaning, data coding or parameter optimisation processes.

\subsection{Description of Machine Learning methods}

Different machine learning approaches have been grouped broadly.

\subsubsection{Gaussian Based Classification}

Gaussian process classifier or GP Classifier[41] considers observations to be drawn from a Bernoulli distribution with probabilities relating to latent functions via sigmoid. Probit transformations define the likelihood model as a function of gaussian cumulative distribution function. In order to account for the non-linearities in the latent space, the covariance function is defined as linear sum of constant, linear, and Matern composite kernels.

\subsubsection{Feature Trimming Method}

The feature trimming method or FT method[33] implements feature set reduction by introducing a random vector in the feature set, calculating the feature importance based on relative mean decrease of Gini-index(derived from random forests) thereby eliminating features scoring less than the dummy variable. Support Vector machines with Gaussian Kernel (RBF-SVM) are further training on the resulting features.

\subsubsection{Distance Weighted Discriminant method}

The distance weighted discrimination(DWD) method[17] is a single base classifier where the high dimension low sample size(HDLSS) property is a subspace in the whole feature space. The HDLSS settings are known to work well 
with generative statistical models that better describe similarity between unobserved multimodal groups. Hence, the linear projection vectors with the data-piling property classify the data and the approach combined with Support Vector machines(SVM) entails accounting for distances from sample to separating hyperplane by associating a misclassification penalty cost $\mathrm{C}(=300)$ to sample points away from the hyperplane. The DWD method also further uses a second-order cone programming(SOCP) optimisation.

\subsubsection{Other methods}

Popular methods include -

(i) $\mathrm{K}$ nearest neighbours 3

(ii) Multiple linear support vector instances 4

amongst others.

\subsection{Automated Machine Learning methods}

The description and evaluation of specified tools show that while automating strenuous aspects of the pipeline can enhance developer productivity, some tools are not yet capable of catering to tasks with little data, or to those with limited domain knowledge.

\subsubsection{Ludwig}

Ludwig is a Tensorflow based, deep-learning, encoder-decoder based toolbox that eliminates the need to write any code. Under the hood, Ludwig performs model training on Horovod[28], open source distributed training framework. Ludwig can generalise across training set types but for structured data, dependencies involve YAML files which allow control over model parameters. Intermediate pre-processed hdf5 file is built along with meta-data that maps input tensors to corresponding labels. During prediction, metadata is loaded to mimic the mapping of new data to its label thereby preventing retraining and re-computation of tensor values. This system of encoding-decoding works well here as the structure of the train data is very similar to that of the test data. The combiner between the encoder-decoder combines features into a single representation.

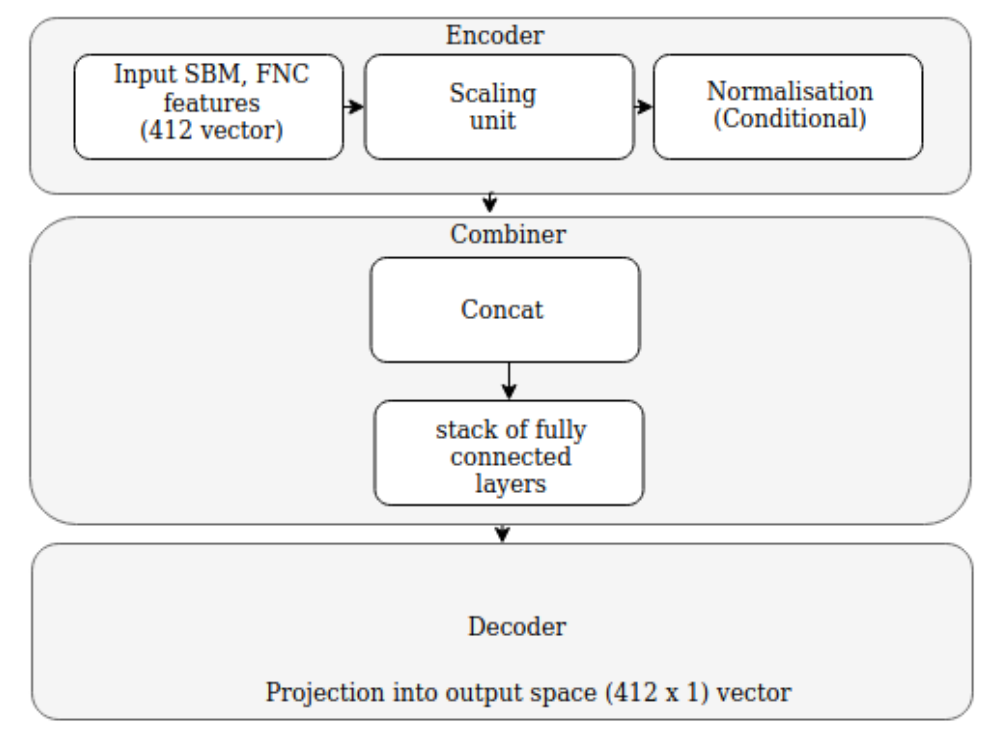

Figure 5: Architecture of Ludwig 5

(a) Default Configuration: Features are automatically converted to a 412-vector and the default model with one encoder(stacked CNN model), one decoder(potentially empty stack of fully connected layers) and one combiner(concatenation by default) finally projects onto a single prediction. The non tensor inputs are reduced to their sums

\footnotetext{
https://github.com/KonstantinTogoi/MLSP2014

4 https://github.com/gabegaster/kaggle_schizophrenia_2014

${ }^{5}$ https://uber.github.io/ludwig/
} 
and the parameters by default are: mean squared error for loss and ReLU activation function with no dropout. Training runs for 100 epochs with Adam optimiser (beta1: 0.9, beta2: 0.999, epsilon: 1e-08), learning rate: 0.001 and decay rate of 0.96 .

(b) Other Configurations: Ludwig does not directly support ensemble modeling. Experimentation with deep learning modules including:

(i) Increasing the number of layers,

(ii) Increasing layer size (from 256 to 512),

(iii) Input normalisation of SBM and FNC features using z-score or minimax,

(iv) Other activation functions like sigmoid and

(v) Dropout regularisation

but these did not lead to substantial performance enhancement.

\subsection{2 $\mathrm{H} 2 \mathrm{O}$}

An in-memory distributed scalable machine learning platform, H2O.ai finds an open port and connects localhost to a $\mathrm{H} 2 \mathrm{O}$ cluster instance. The internal architecture of $\mathrm{H} 2 \mathrm{O}$ is as illustrated in Figure 6.

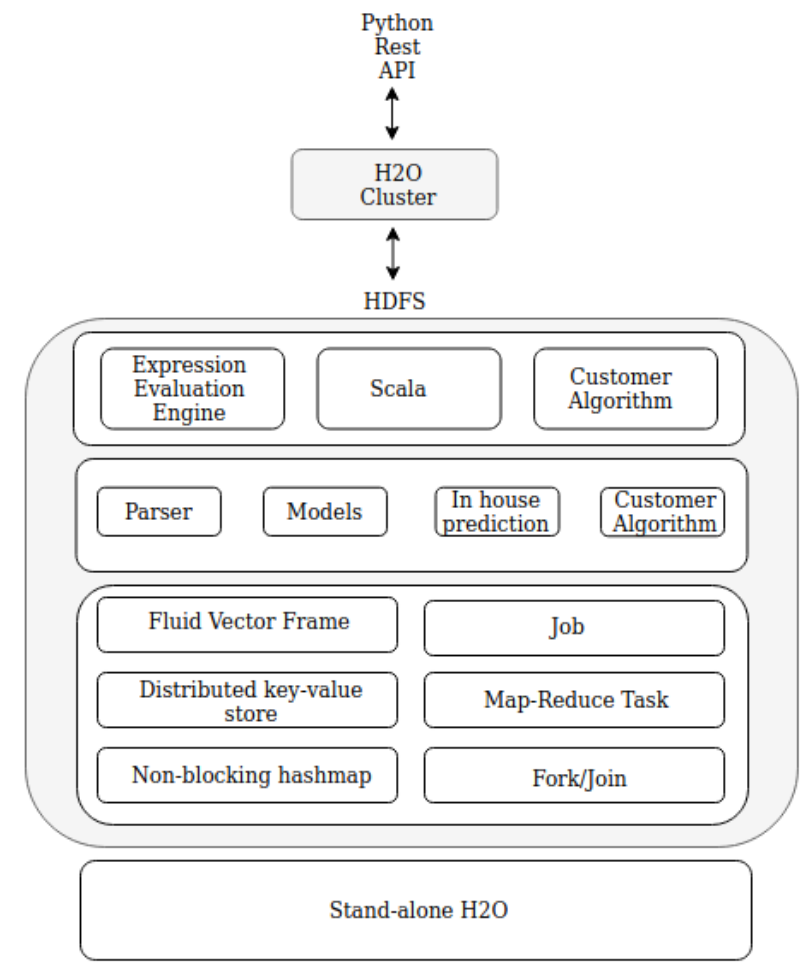

Figure 6: Architecture of $\mathrm{H}_{2} \mathrm{O}^{6}$

(a) Default H2O.ai Configuration: The default $\mathrm{H} 2 \mathrm{O}$ configuration accounts for the input data via 5 fold cross validated model performance. The framework performs neural architecture search optimising for the AUROC metric and ranks all obtained algorithms on a leaderboard as per training performance which in this case includes: gradient based methods, stacked ensembles, generalized linear models, distributed random forests and XGBoost frameworks respectively.

(b) H2O.ai deeplearning: H2O's deep learning module is based on a multi-layer feed-forward artificial neural network (multilayered perceptron) trained with stochastic gradient descent using back propagation. Each computational node trains a copy of the global model's parameters with asynchronous multi-threading and contributes across the network through periodic model averaging. The different experimentation settings with the deep learning module include:

(i) Activation functions - tanh, ReLU, maxout

(ii) Regularisation functions - dropout

\footnotetext{
${ }^{6}$ http://docs.h2o.ai/h2o/latest-stable/h2o-docs/architecture.html
} 
(iii) Categorical encoding methods - one hot internal encoding: N+1 new columns for categorical features with N levels, and sort by response encoding: reordering of levels according to mean response. (c) Other configurations: Ensemble and stacking did not yield substantial results.

\subsubsection{ATM - Autotune models}

This multi-user, multi-data AutoML framework allows an end-to-end automated neural pipeline to search for the best model and henceforth train the best found algorithm like a normal sklearn model which is then pickled to classify on new data. The architecture of ATM is as follows:

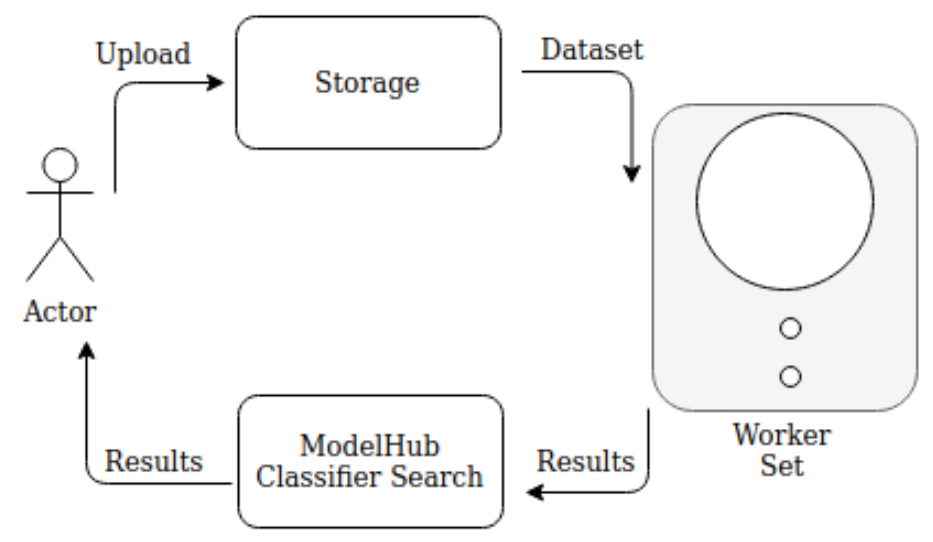

Figure 7: Architecture of ATM[26]

(a) Default Configuration: In the default configuration, search space of 100 classifiers is explored. The best performing model is a K-Nearest Neighbor $(\mathrm{KNN})$ with the ball tree algorithm $(\mathrm{k}=42)$. Default models in queue include: logistic regression, decision trees and variants of KNN.

(b) Other Configurations: Increasing the budget of the classifiers leads to exploration of variants of KNN with different $\mathrm{k}$ values but does not necessarily lead to better performance on the private set. Using other methods like support vector machines (SVM), stochastic gradient descent (SGD), Gaussian and multinomial naive Bayes (NB), random forests, multi-layer perceptrons (MLP) and boosting does not improve performance significantly.

\subsubsection{AutoGluon-Tabular}

This automated framework offers multi-layer ensembling that is trained layer-wise to translate raw data into predictions. Skip connections in stack ensembling and neural network embedding as well as repeated k-fold bagging are employed to curb overfitting. AutoGluon-Tabular is also capable of inferring properties of the prediction task like variable types and problem class. The architecture of AutoGluon-Tabular is as illustrated in Figure 8.

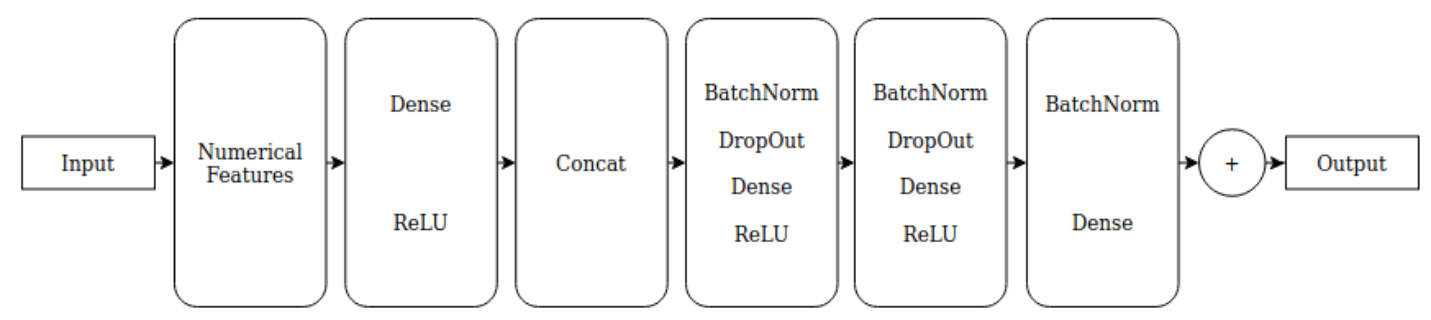

Figure 8: Architecture of AutoGluon[24]

(a) Default Configuration: AutoGluon by default enables prototyping of deep learning systems with automatic hyperparameter tuning, model selection and data processing while choosing a random training-validation data split with conditional stratified sampling strategies. Since the described methods are computationally intensive, the process is 
parallelised across threads and machines (depending on amount of available distributed resources). However, AutoGluon opts for tuning on validation data using internal knobs and hence, internal performance estimated maybe higher as compared to private or public AUC. Predictions are auto-optimised to reduce inference latency.

(b) Other configurations: Bagging, stacking or ensembling did not lead to substantial performance enhancements.

\subsubsection{Box}

MLBox is an automated machine learning framework that uses drift scores to select important feature attributes by discarding variables automatically that have distributions that do not align with the rest of the data(covariate shift) and therefore, do not contribute to the prediction process which in turn also enhances performance on biased data by relating the ROC score as measure of drift. If the drift is high, then the sets of data are discernible and if low, they are not. MLBox implicitly uses a drift thresholder that retains only features lesser than the threshold. The architecture is as shown in Figure 9.

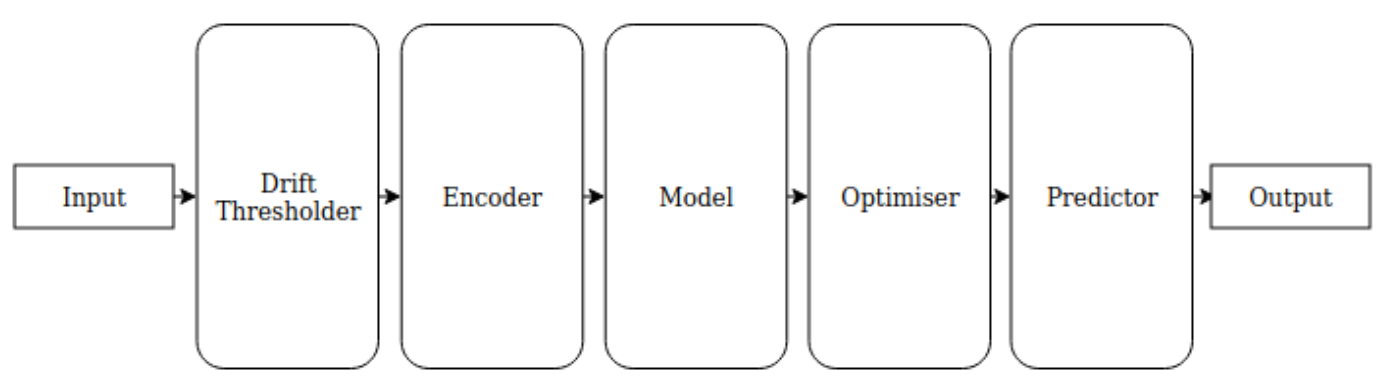

Figure 9: Architecture of ML Box!

(a) Default configuration: There is no default configuration of MLBox for the space search. So, the following space configurations were evaluated:

(i) A stacking classifier with multiple Adaboost classifiers

(ii) Encoding strategy with random projections, label encodings and entity embeddings. Feature selection threshold with uniform search in the $[0.001,0.2]$ space. Final estimation strategy in the random forests, extra trees and light gradient based methods space.

(b) Other configurations: Widening the search space does not necessarily lead to better private AUC performance.

\subsubsection{AutoML-gs}

AutoML-g $\AA^{8}$ generates raw Python code using Jinja templates and trains the statistical model in a subprocess by trying differentiating hyperparameters to find the best model. It automatically infers feature types and then uses a ETL strategy for feature optimisation according to their hyperparameters. Encoders used are stored as serialised JSON files with consistent, discrete hyperparameter values instead of uniform hyperparameter values to accurately gauge tuning impact. The architecture is as described in Figure 10.

(a) Default Configuration: There are two environments available in AutoML-gs: Tensorflow and XGBoost. Experimentation has been carried out on both here.

\section{Results}

The results from the mentioned models and configurations are evaluated broadly based on the "area under the receiver operating characteristics curve" (AUROC) statistic under the following subcategories. The ROC represents a probabilistic curve, and AUC represents degree of separability. Higher the statistic, better the model is at segregating schizophrenics against non-schizophrenics.

\footnotetext{
${ }^{7} \mathrm{https}: / /$ mlbox.readthedocs.io/en/latest/

${ }^{8}$ urlhttps://mljar.com/blog/automl-software-list/

${ }^{9}$ https://github.com/minimaxir/automl-gs
} 
A PREPRINT - JANUARY 5, 2021

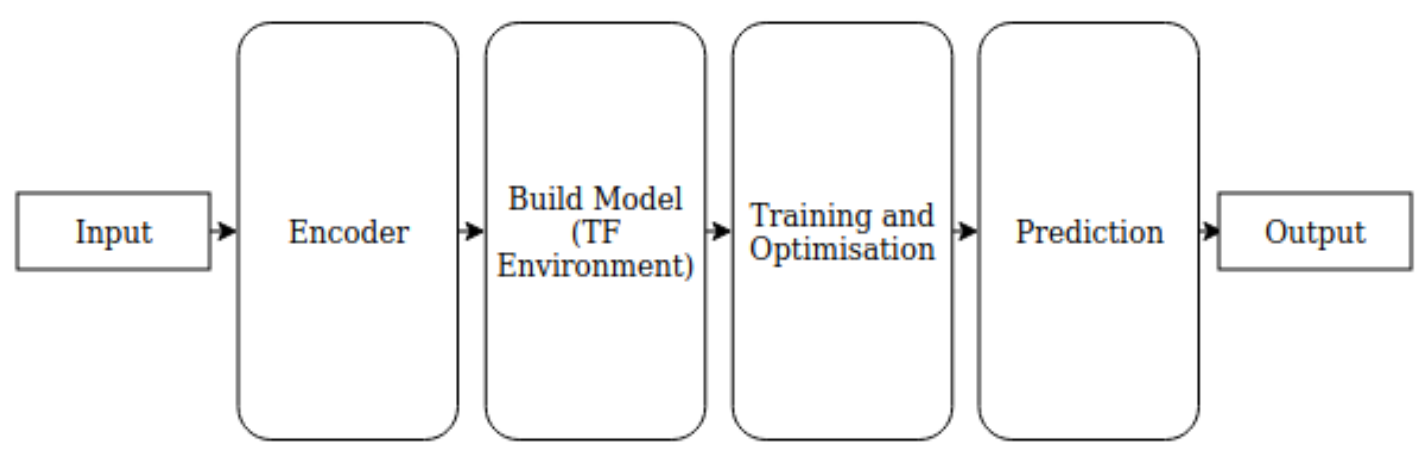

Figure 10: Architecture of AutoML-g s9

The tools and frameworks are compared on the following parameters for the best performing configuration within each tool: (a) Public AUC which involves evaluation of about one percent of the given data. (b) Private AUC which involves evaluation of about 99 percent of (hidden) test data. (c) Overall AUC which involves evaluation on all test data. (d) Model Stability and Robustness (Model-SR) which is defined based on private and public AUC. (e) Interpretability.

It was observed that the overall AUC led to more reasonable assessments about model quality than any other metric[27]. Note: Since private data involves most of test data, public data has not been a priority for performance or optimisation.

The default configuration mode in each tool performs as tabulated in Table 1.

Table 1: Comparison of Default configurations of AutoML methods

\begin{tabular}{lll}
\hline Tool & Public AUC & Private AUC \\
\hline Ludwig & 0.42867 & 0.60000 \\
H2O & 0.75892 & 0.60512 \\
ATM & 0.50892 & 0.48461 \\
AutoGluon & 0.79910 & 0.81025 \\
MLBox & 0.73437 & 0.79487 \\
AutoML-gs & 0.64732 & 0.84615
\end{tabular}

The visual analysis of the data is as shown in Figure 11.

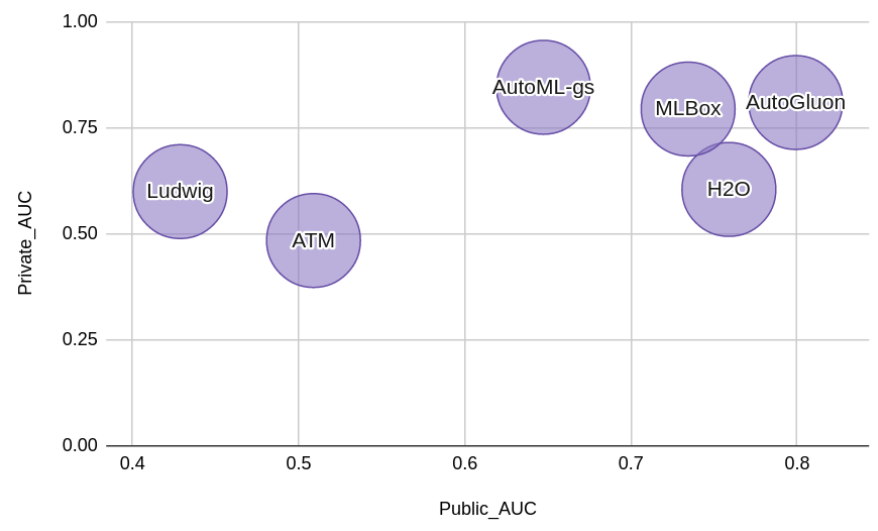

Figure 11: Relative performance of AutoML frameworks: Default configuration

The performance of 2087 entries from 291 participants[27] has been aggregated into the visualisation below in terms of public and private AUC. 
A PREPRINT - JANUARY 5, 2021

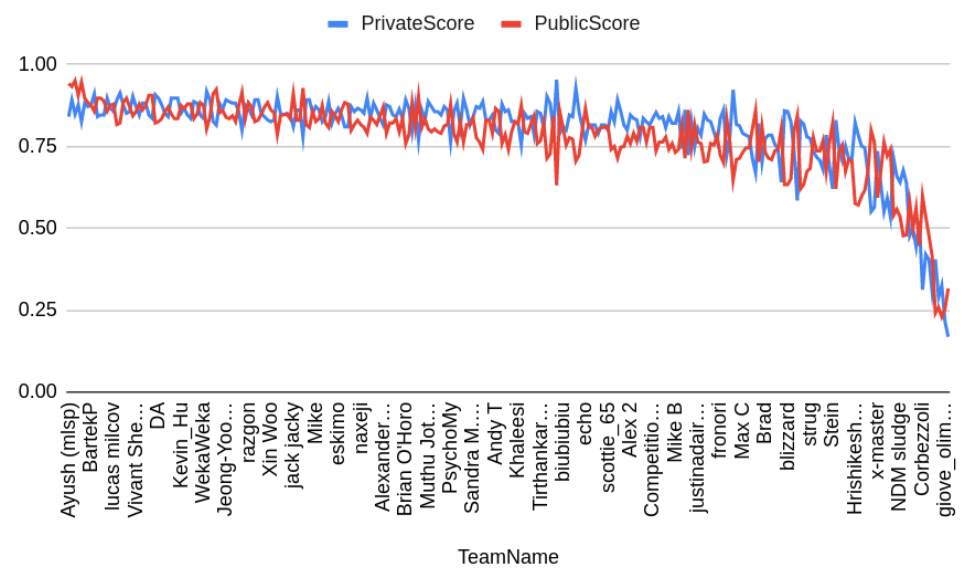

Figure 12: Relative performance of ML frameworks

\subsection{Public AUC}

Most of machine learning methods perform well on private data, but do not perform nearly as well on the public data with the exception of SVM.

Table 2: Relative Performance of Machine Learning methods: Public AUC

\begin{tabular}{ll}
\hline Method & Public AUC \\
\hline GP Classifier & 0.70536 \\
FT Method & 0.64732 \\
DWD Method & 0.84375 \\
\hline
\end{tabular}

However, the best performance on the public data is 0.95758 and the highest scoring AutoML model - one hot encoding of categorical features with rectifier on $\mathrm{H} 2 \mathrm{O}$ - is 0.95384 . This score has not been optimised further here as our obtained performance is already at the top 1 percent of all models.

Table 3: Relative Performance of AutoML methods: Public AUC

\begin{tabular}{lll}
\hline Method & Sub-configuration & Public AUC \\
\hline Ludwig & 512 FC layers & 0.55357 \\
H2O & ReLU activation & 0.88839 \\
H2O & sortbyResponse encoding with dropout & 0.80803 \\
H2O & OneHotInternal encoding with rectifier & 0.95384 \\
AutoGluon & Default & 0.79910 \\
MLBox & Default & 0.73437
\end{tabular}

The performance of all models is as shown in Figure 13 where the y-axis represents the Public AUC score and x-axis represents the model. $\mathrm{H} 2 \mathrm{O}$ SR refers to the $\mathrm{H} 2 \mathrm{O}$ sort by response encoding with dropout configuration and $\mathrm{H} 2 \mathrm{O} 1 \mathrm{HiE}$ refers to the $\mathrm{H} 2 \mathrm{O}$ one hot internal encoding with rectifier configuration.

\subsubsection{Private AUC}

Gradient based methods seem to perform the best in terms of generalisation as they account for intricacies including short-scale, non-linearities in the latent space while maintaining model flexibility. It is notable that - most of these automated machine learning tools lean towards gradient based classifiers similar to developer behavior[27]. 


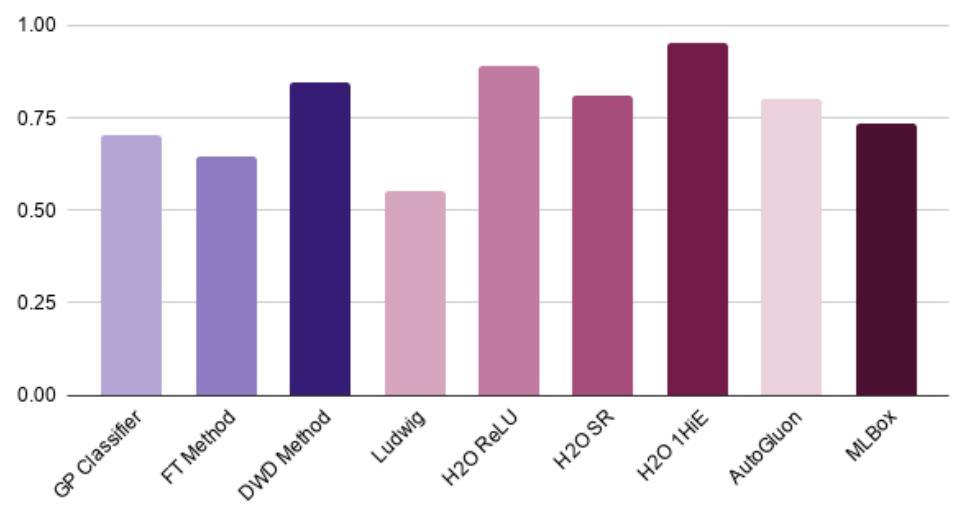

Figure 13: Relative Performance of ML and AutoML methods: Public AUC

Table 4: Relative Performance of Machine Learning methods: Private AUC

\begin{tabular}{ll}
\hline Method & Private AUC $(\mu \mathrm{m})$ \\
\hline GP Classifier & 0.92820 \\
FT Method & 0.92307 \\
DWD Method & 0.91282 \\
\hline
\end{tabular}

Table 5: Relative Performance of AutoML methods: Private AUC

\begin{tabular}{lll}
\hline Method & Sub-configuration & Private AUC \\
\hline Ludwig & Default & 0.60000 \\
H2O & Gradient-based & 0.72307 \\
ATM & Classifiers=500 & 0.83333 \\
AutoGluon & Default & 0.6923 \\
MLBox & Default & 0.73437 \\
AutoML-gs & Default & 0.82820 \\
\hline
\end{tabular}

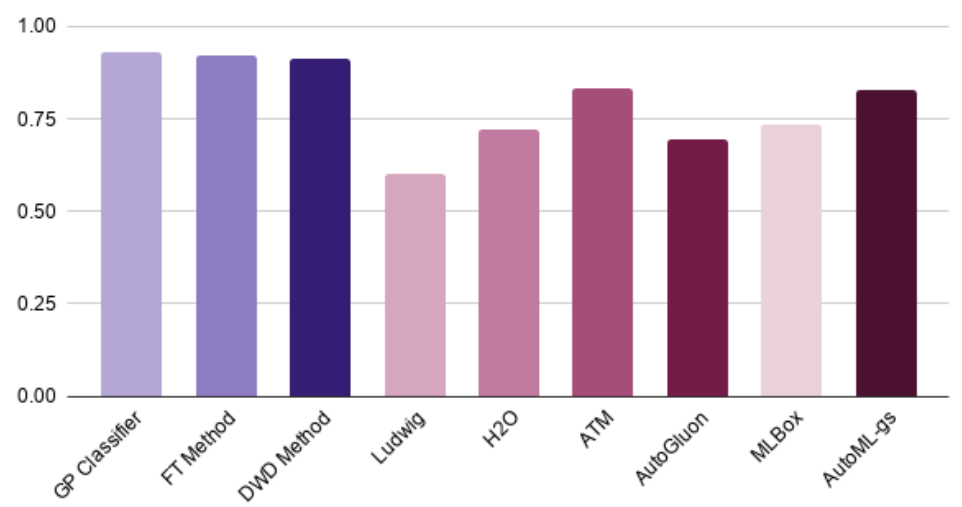

Figure 14: Relative Performance of ML and AutoML methods: Private AUC

On further experimentation with H2O's deep learning module configurations, the following observations were made:

(a) ReLU generalised better with dropout regularisation.

(b) Models with categorical encoding tended to perform best with respect to Private AUC. 
bioRxiv preprint doi: https://doi.org/10.1101/2020.11.02.364976; this version posted January 5, 2021. The copyright holder for this preprint (which was not certified by peer review) is the author/funder, who has granted bioRxiv a license to display the preprint in perpetuity. It is made available under aCC-BY-NC-ND 4.0 International license.

A PREPRINT - JANUARY 5, 2021

Table 6: Relative Performance of $\mathrm{H} 2 \mathrm{O}$ deep learning configurations: Private AUC

\begin{tabular}{ll}
\hline H2O Sub-configuration & Private AUC \\
\hline Tanh activation & 0.88717 \\
ReLU + dropout & 0.85641 \\
Maxout & 0.91794 \\
Maxout + dropout & 0.91282 \\
SoftResponseEncoding Rectifier + dropout & 0.94358 \\
\hline
\end{tabular}

H2O deep learning configured models perform comparably to ML models.

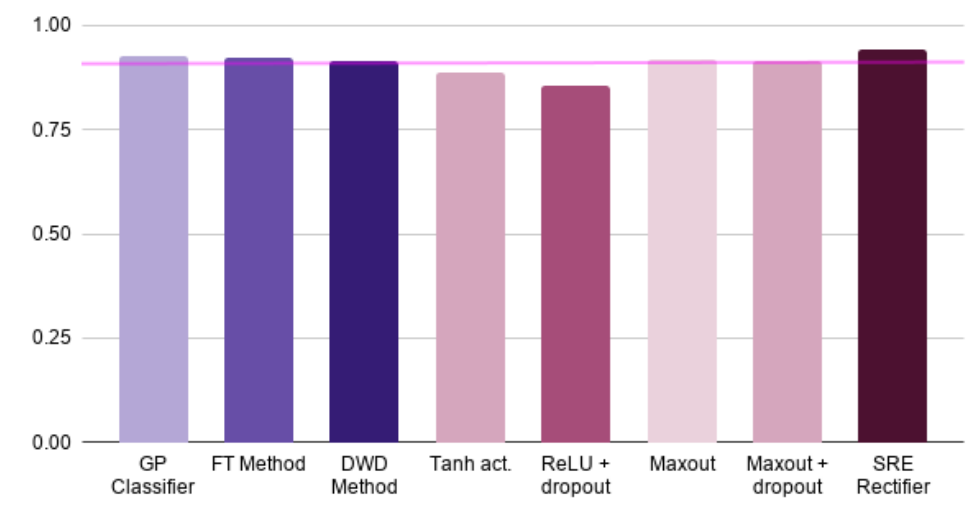

Figure 15: Relative Performance of H2O and ML methods: Private AUC

\subsubsection{Overall AUC}

The Overall AUC helps favor consistent models while ensuring retention of generalisability. However, none of the machine learning models have an Overall AUC of $>0.9[27]$ which is hereby known as "the 0.9 wall". The best models in this respect average to about 0.88 but this is an important reinforcement of the performance of machine learning classifiers in challenging settings. It is also worthwhile to consider the fact that although this result is obtained with features derived from independent components using spatio-temporal regression highlighting the predictive utility of imaging data, the identification of appropriate predictive features within the entire space of features is still a challenging process.

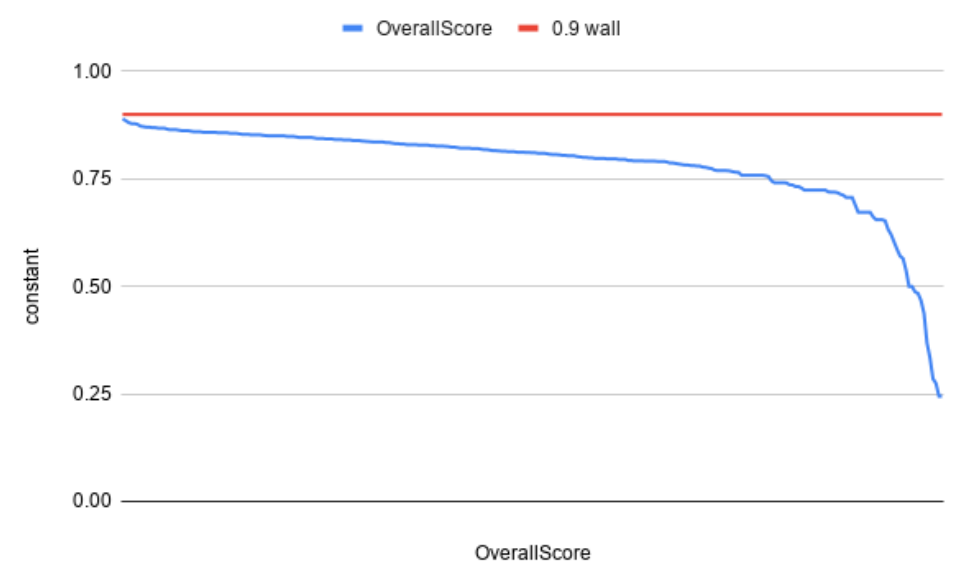

Figure 16: Relative performance of all machine learning models( 290): Overall AUC 
Most machine learning methods work better than the benchmark average with SVM based algorithms performing the best.

Table 7: Relative Performance of Machine Learning methods: Overall AUC

\begin{tabular}{llll}
\hline Method & Private AUC & Public AUC & Overall AUC \\
\hline GP Classifier & 0.92820 & 0.70536 & 0.79785 \\
FT Method & 0.92307 & 0.64732 & 0.75862 \\
DWD Method & 0.91282 & 0.84375 & 0.86206 \\
\hline
\end{tabular}

AutoML tools automate the process of feature identification efficiently even with little training data and high dimensional features. Table 15 highlights that subtle configuration changes such as changing categorical encoding methodology or activation functions can comfortably scale the 0.9 wall.

Table 8: Relative Performance of AutoML methods: Overall AUC

\begin{tabular}{llll}
\hline Method & Private AUC & Public AUC & Overall AUC \\
\hline $\mathrm{H} 2 \mathrm{O}$ with activation & 0.91794 & 0.88839 & 0.9 \\
$\mathrm{H} 2 \mathrm{O}$ encoding with ReLU activation & 0.94358 & 0.95384 & $\mathbf{> 0 . 9}$ \\
\hline
\end{tabular}

The aggregate Overall AUC of the data is as shown.

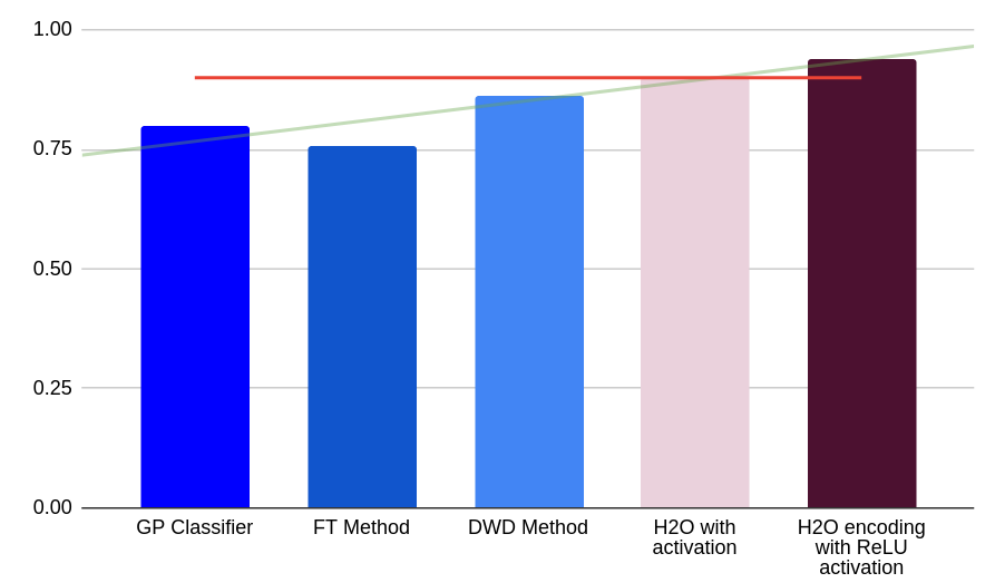

Figure 17: Relative Performance of ML and AutoML methods: Overall AUC

\subsubsection{Model Stability and Robustness(Model-SR)}

Model stability and robustness which we define here as Model-SR is :

ModelSR $=\mid$ PublicAUC - PrivateAUC $\mid$

and a model is said to be stable if this absolute difference is nearly 0 . However this does not seem to be the case with many machine learning models. The Model-SR calculated from 290 models is as shown in Figure 18. 


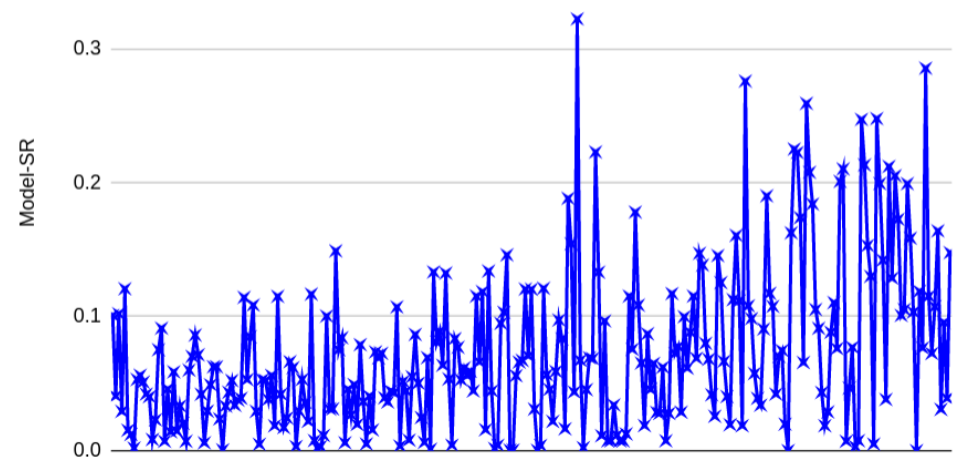

Figure 18: Relative performance of ML models ( 290): Model-SR

Amongst the models that perform best with respect to private AUC, Gradient based methods show the highest degree of variability with Model-SR as even observed with Gradient based models from Automated tools. Support vectors models display maximum stability.

Table 9: Relative Performance of Machine Learning methods: Model-SR

\begin{tabular}{llll}
\hline Method & Private AUC & Public AUC & Model-SR \\
\hline GP Classifier & 0.92820 & 0.70536 & 0.22284 \\
FT Method & 0.92307 & 0.64732 & 0.27575 \\
DWD Method & 0.91282 & 0.84375 & 0.06907 \\
\hline
\end{tabular}

The effect of stability of automated tools is observed from 132 different configurations of more than six different tools on multimodal schizophrenia data. The most stable AutoML models are AutoGluon followed by MLBox and H2O (default configuration). Deep learning $\mathrm{H} 2 \mathrm{O}$ modules with eigen encoding and maxout display the maximum stability at 0.01921 while AutoML-gs and ludwig seem to display the least stability.

Table 10: Relative Performance of AutoML methods: ModelSR

\begin{tabular}{ll}
\hline Tool:Configuration & Difference AUC \\
\hline AutoGluon: default & 0.00927 \\
MLBox: default & 0.0652 \\
ATM: 500 classifier ${ }_{b}$ udget & 0.10119 \\
H2O: default & 0.0445 \\
H2O: activation & 0.18 \\
H2O: ReLU + dp & 0.18384
\end{tabular}


A PREPRINT - JANUARY 5, 2021

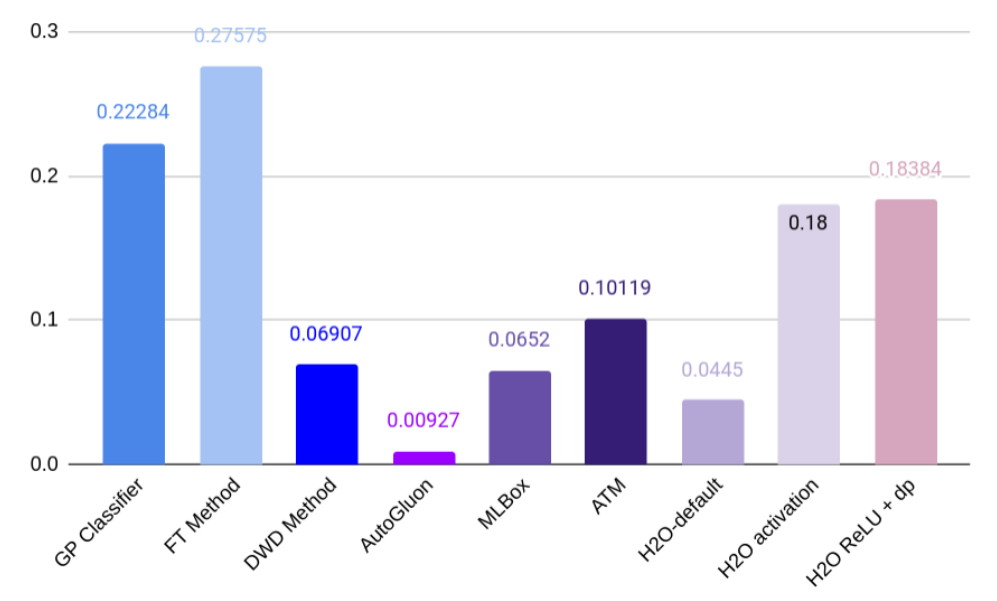

Figure 19: Relative performance of ML vs AutoML models: Model-SR

\subsubsection{Interpretability}

AutoML tends to further obscure black-boxed models. But interpretability is of utmost value for healthcare diagnostics and for other safety critical applications in general. While most of the tools used in the study enable external augmentations and interpretable enhancements, few internally support interpretability.

MLBox: MLBox has an internal prediction feature that highlights the top features as per their drift coefficients that most influence the output.

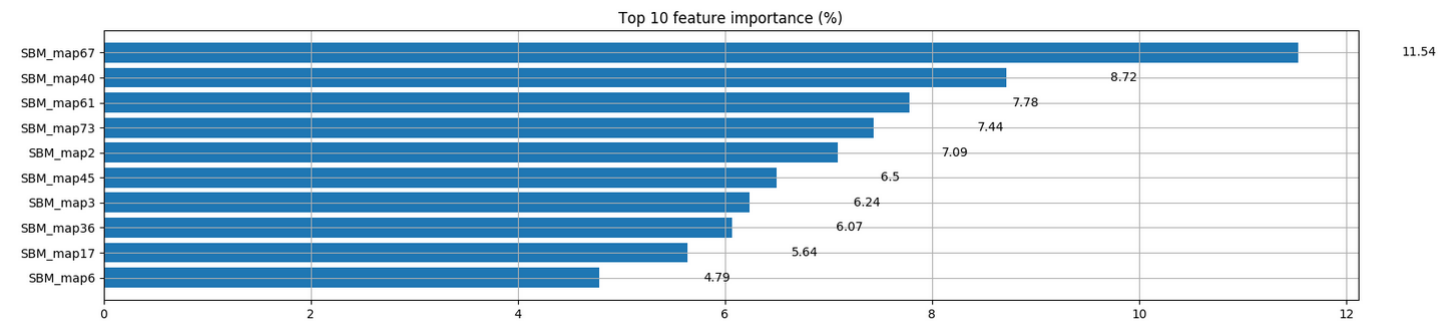

Figure 20: Relative Feature importance in MLBox

AutoML-gs: The output predictions are shipped along with json files containing the mean, scale and variance of every feature in the dataset that the encoder model is trained on. It also contains additional information about the training time taken, the performance parameters including - log loss, accuracy, AUC, precision, recall and f1 score for every epoch. The model weights along with the entire pipeline can be accessed and manipulated. This makes this AutoML framework nearly equivalent to any machine learning framework.

H2O: In case of deep learning, hidden model parameters, variable importance, graphs, weights/biases, and the status of neuron layers namely - layer number, units, type, dropout, L1 regularisation, L2 regularisation, mean rate, rate root-mean-square, momentum, mean weight, weight root-mean-square, mean bias, and bias root-mean-square are logged. Visualisations can be built externally with Tableau.

Ludwig: Since the definitions are externally specified through the YAML structure, interpretability with respect to feature engineering lies much outside the framework than within. Experimental runs are saved along with predictions, probabilities, and different classes of statistics including: (A) Class statistics which includes confusion matrix, average precision across samples and (B) Performance related metrics including loss, accuracy, F1, kappa score, token accuracy, precision, and recall (C) Per class statistics for all True/False values - fallout, discovery rates, false negative rates, omission rates, false positives, hit rate, informedness, markedness, matthew's correlation coefficient, miss-rate, predictive values, specificity, sensitivity, true negative rate, and true positive rate. It also accounts for logging model hyperparameters for each feature, indexing, checkpointing, tracking training progress, and working with meta data. These parameters can be visualized externally via Tensorboard. 
ATM: Each of the models and model parameter metrics in the range of the classifier budget are saved for later access. The framework is nearly equivalent to other machine learning models in terms of interpretability.

AutoGluon: Models generated are saved along with information about existing and generated features. On running this instance, the dask worker space is first locked before start of training. Visualizations can built externally via tensorboard or mxboard.

\section{Conclusion and Scope for future work}

Automated machine learning tools have a remarkable ability to effectively deal with multimodal data under high dimension low sample size settings(HDLSS) as demonstrated. The space of configurations and frameworks has transformed over the years but the generalisability of these methods to raw or lightly processed data and their ability to automatically identify features from whole brain information in a clinical setting still remains a challenge. With respect to healthcare systems in particular, there is a need to develop automated frameworks that are not only consistent across executions for reproducibility, but also more transparent, ethical, and interpretable. Future research in this direction would hence require tools that better understand data, and hence are able to employ reasonable choice of algorithmic models along with correspondingly different tuning strategies.

\section{Acknowledgements}

(i) Data collection (partially described in [19]), available on Kaggle 10 , was performed at the Mind Research Network, and funded by a Center of Biomedical Research Excellence (COBRE) grant 5P20RR021938/P20GM103472 from the NIH to Dr. Vince Calhoun.

(ii) The suggestions of Erin LeDell (H2O.ai), @ PGijsbers(gama), Piero Molino (Ludwig), Dr. V Krishnamurthy, Dr. S Natarajan, Anil B Murthy and Alex De Romblay (MLBox).

\section{References}

[1] Lena Palaniyappan et al Voxel-Based Morphometry for Separation of Schizophrenia From Other Types of Psychosis in First-Episode Psychosis: Diagnostic Test Review In Schizophrenia Bulletin, Oxford Academic, Volume 42, Issue 2, March 2016, pages 277-278.

[2] K Ohi et al. Structural Alterations of the Superior Temporal Gyrus in Schizophrenia: Detailed Subregional Differences In European psychiatry: the journal of the Association of European Psychiatrists, National Center for Biotechnology Information(NCBI), May 2016, 35:25-31.

[3] D Gupta. Limbic Systems. In Essentials of Neuroanesthesia, Neuroanatomy, Science Direct, March 2017, pages 3-40.

[4] Lei Chu et al Individual Recognition in Schizophrenia using Deep Learning Methods with Random Forest and Voting Classifiers: Insights from Resting State EEG Streams In arXiv:1707.03467v2 [cs.CV], January 2018.

[5] Metea Nikolac Perkovic et al. Theranostic biomarkers for Schizophrenia. In International journal of molecular sciences, National Center for Biotechnology Information, 2017, pages 733.

[6] Pieter Gijsbers et al. An Open Source AutoML Benchmark. In ICML Workshop on Automated Machine Learning, 2019.

[7] Hsin-Hui Huang et al. Factors in Maintaining a Stable Patient-Physician Relationship Among Individuals with Schizophrenia In Community Mental Health Journal, National Center for Biotechnology Information, March 2017.

[8] Suji Ham et al. Drug Abuse and Psychosis: New Insights into Drug-induced Psychosis In Experimental neurobiology, National Center for Biotechnology Information, Febraury 2017, 26(1): 11-24.

[9] Po-Han Chou et al Duration of Untreated Psychosis and Brain Function during Verbal Fluency Testing in FirstEpisode Schizophrenia: A Near-Infrared Spectroscopy Study In Scientific Reports 5, 18069, Nature, December 2015.

[10] Paula Moran et al. Gene Environment Interactions in Schizophrenia: Evidence from Genetic Mouse Models. In Journal of neural transplantation and plasticity, National Center for Biotechnology Information, 2016.

\footnotetext{
10 https://www.kaggle.com/c/mlsp-2014-mri/
} 
[11] Shu Lih Oh et al. Deep Convolutional Neural Network Model for Automated Diagnosis of Schizophrenia Using EEG Signals In Machine Learning for Biomedical Data Analysis, Multidisciplinary Digital Publishing Institute(MDPI), July 2019.

[12] Mohammad R Arbabshirani et al. Classification of schizophrenia patients based on resting-state functional network connectivity In Frontiers in Neuroscience, July 2013.

[13] Xin He et al. AutoML: A Survey of the State-of-the-Art In Arxiv 1908.00709, 2020.

[14] Adithya Balaji et al. Benchmarking Automatic Learning Frameworks. In Arxiv 1808.06492, 2018.

[15] Marc-Andre Zoller et al. Benchmark and Survey of Automated Machine Learning Frameworks. In Arxiv 1904.12054, 2020.

[16] Krishna R Patel et al. Schizophrenia: Overview and Treatment Options In Pharmacy and Therapeutics, National Center for Biotechnology Information, 638-645, 2014.

[17] Karolis Koncevicius et al. The 10th annual MLSP competition: Third Place In IEEE International Workshop on Machine Learning for Signal Processing(MLSP), September 2014.

[18] Matthew Glasser et al. The Human Connectome Project's neuroimaging approach In Nature Neuroscience 19, 1175-1187, 2016.

[19] M. Cetin, F. Christensen, C. Abbott, J. Stephen, A. Mayer, J. Canive, J. Bustillo, G. Pearlson, and V. D. Calhoun. Thalamus and posterior temporal lobe show greater inter-network connectivity at rest and across varying sensory loads in schizophrenia. In NeuroImage, in press.

[20] Elena A Allen et al. Tracking Whole-Brain Connectivity Dynamics in the Resting State In Cerebral Cortex, Oxford Academic, Volume 24, Issue 3, Pages 663-676, 2014.

[21] Segall JM et al. Correspondence between structure and function in the human brain at rest. In Frontiers in NeuroInformatics, National Center for Biotechnology Information, 2012.

[22] Piero Molino et al. Ludwig: a type-based declarative deep learning toolbox In arXiv: 1909.07930, 2019.

[23] Candel A et al. Deep learning with H2O. url: http://docs.h2o.ai/h2o/latest-stable/h2odocs/booklets/DeepLearningBooklet.pdf In 2020.

[24] Erickson et al. AutoGluon-Tabular: Robust and Accurate AutoML for Structured Data In arXiv preprint:2003.06505, 2020.

[25] Axel Aronio De Romblay et al. MLBox. url: https://mlbox.readthedocs.io/en/latest/installation.html In 2017.

[26] Thomas Swearingen et al. ATM: A distributed, collaborative, scalable system for automated machine learning. In IEEE BigData 2017, 151-162

[27] Rogers F Silva et al. The Tenth Annual MLSP Competition: Schizophrenia Classification Challenge In IEEE International Workshop on Machine Learning for Signal Processing, September 2014.

[28] Alexander Sargeev et al. Horovod: fast and easy distributed deep learning in TensorFlow In arXiv preprint arXiv: 1802.05799, 2018.

[29] Evrim Acar et al Unraveling Diagnostic Biomarkers of Schizophrenia Through Structure-Revealing Fusion of Multi-Modal Neuroimaging Data In Frontiers of Neuroscience, May 2019.

[30] Piotr Stowinski et al Unraveling socio-motor biomarkers in Schizophrenia In npj Schizophr, Nature, 2017.

[31] Selwyn B Renard et al Unique and Overlapping Symptoms in Schizophrenia Spectrum and Dissociative Disorders in Relation to Models of Psychopathology: A Systematic Review In Schizophr Bull, National Center for Biotechnology Information, 118-121, 2017.

[32] Yujia Zhang et al A review of autobiographical memory studies on patients with schizophrenia spectrum disorders In BMC Psychiatry 19, 361, Springer Nature, 2019.

[33] Alexander V Lebedev The 10th annual MLSP competition: Second place In IEEE International Workshop on Machine Learning for Signal Processing(MLSP), September 2014.

[34] Katherine G. Jonas et al Lead-Time Bias Confounds Association Between Duration of Untreated Psychosis and Illness Course in Schizophrenia In Americal Journal of Psychiatry, 2020.

[35] Hongyun Qin et al Duration of untreated psychosis and clinical outcomes of first-episode schizophrenia: a 4-year follow-up study In Shanghai Arch Psychiatry, National Center for Biotechnology Information (NCBI), 26(1):42-48, February 2014.

[36] Folorunsho Tajudeen Nuhu et al Strong family history and early onset of schizophrenia: about 2 families in Northern Nigeria In Pan Africal Medical Journal, National Center for Biotechnology Information, 24: 282, 2016. 
A PREPRINT - JANUARY 5, 2021

[37] E. B. Erhardt, et al Comparison of Multi-Subject ICA Methods for Analysis of fMRI Data In Human Brain Mapping, vol.32, no.12, pp. 2075-2095, 2011.

[38] J. M. Segall et al Schizophrenia: Voxel-based morphometric multisite collaborative study on schizophrenia In Schizophr Bull, vol. 35, no. 1, pp. 82-95, 2009.

[39] Chao Deng et al Mapping the pathophysiology of schizophrenia: interactions between multiple cellular pathways In Frontiers in Cellular Neuroscience, November 2013

[40] Paul Allen et al Emerging Temporal Lobe Dysfunction in People at Clinical High Risk for Psychosis In Frontiers in Psychiatry - Schizophrenia, May 2019

[41] Arno Solin et al The 10th annual MLSP competition: First place In IEEE International Workshop on Machine Learning for Signal Processing (MLSP), September 2014.

[42] Assen Jablensky, MD The diagnostic concept of schizophrenia: its history, evolution, and future prospects In Dialogues in Clinical Neuroscience, National Center for Biotechnology Information, Sept 2010.

[43] Srivathsan Srinivasagopalan et al A deep learning approach for diagnosing schizophrenic patients, In Journal of Experimental Theoretical Artificial Intelligence, 2019.

[44] Jihoon Oh et al Identifying Schizophrenia Using Structural MRI With a Deep Learning Algorithm In Frontiers in Psychiatry, 2020.

[45] Ling-Li Zeng et al Multi-Site Diagnostic Classification of Schizophrenia Using Discriminant Deep Learning with Functional Connectivity MRI In EBioMedicine, The Lancet, 30 74-85, 2018.

[46] Cynthia S Weickert et al Biomarkers in Schizophrenia: A Brief Conceptual Consideration In Disease markers, National Center for Biotechnology Information (NCBI), 35(1):3-9, 2013.

[47] Vivek Bambole et al Symptom overlap between schizophrenia and bipolar mood disorder: Diagnostic issues In Open Journal of Psychiatry, 3(04):8-15, 2013.

[48] Stefano Pallanti et al Raising attention to attention deficit hyperactivity disorder in schizophrenia In World Journal of Psychiatry, 5(1): 47-55, March 2015.

[49] R Schmidt-Kastner et al An environmental analysis of genes associated with schizophrenia: hypoxia and vascular factors as interacting elements in the neurodevelopmental model In Molecular Psychiatry 17, 1194-1205, January 2012.

[50] Jessica A Turner et al Heritability of Multivariate Gray Matter Measures in Schizophrenia In Twin research and human genetics, National Center for Biotechnology Information (NCBI), 15(3):324-35, June 2012.

[51] Cota Navin Gupta et al Patterns of Gray Matter Loss in Schizophrenia from a Large-Scale Aggregated Dataset In Schizophrenia Bulletin, 2015.

[52] Eduardo Castro et al Identification of Patterns of Gray Matter Abnormalities in Schizophrenia Using Source-Based Morphometry and Bagging In 36th EMBC, Chicago, IL, 2014, In Press.

[53] Sergey M. Plis et al Deep learning for neuroimaging: a validation study In arXiv:1312.5847, February 2014.

[54] Mireille Nieuwenhuis et al Classification of Schizophrenia Patients and Healthy Controls From Structural MRI Scans in Two Large Independent Samples In Neuroimage, National Center for Biotechnology Information (NCBI), 61(3):606-12, July 2012.

[55] Paul H Lysaker et al Metacognitive function and fragmentation in schizophrenia: Relationship to cognition, self-experience and developing treatments In Schizophrenia Research: Cognition, Elsevier, Volume 19, March 2020..

[56] Carl M Sellgren et al Increased synapse elimination by microglia in schizophrenia patient-derived models of synaptic pruning In Nature Neuroscience, Volume 22, 374-385, $2019 .$.

[57] I-Jun Chou et al Familial Aggregation and Heritability of Schizophrenia and Co-aggregation of Psychiatric Illnesses in Affected Families In Schizophr Bull, 43(5), 1070-1078, September 2017.

[58] Benjamin JS al-Haddad MD et al The fetal origins of mental illness In Americal Journal of Obstetrics and Gynecology, Elsevier, Volume 221, Issue 6, Pages: 549-562, December 2019..

[59] Irina Rish et al Functional Network Disruptions in Schizophrenia In Methods in Molecular Biology, National Center for Biotechnology Information, 1613, 479-504, $2017 .$.

[60] V. D. Calhoun, and T. Adali, Multisubject Independent Component Analysis of fMRI: A Decade of Intrinsic Networks, Default Mode, and Neurodiagnostic Discovery In IEEE Reviews in Biomedical Engineering, vol. 5, pp. 60-73, 2012. 